\title{
¿Buscando al culpable? La estrategia discursiva en Twitter de los actores políticos populistas europeos en tiempos de crisis ${ }^{1}$
}

Looking for the Guilty? The Discursive Strategy on Twitter of the European Populist Political Actors in Times of Crisis

LAURA ALONSO-MUÑOZ

UNIVERSITAT JAUME I DE CASTELLÓ

HTTPS://ORCID.ORG/0000-0001-8894-1064

ANDREU CASERO-RIPOLLÉS

UNIVERSITAT JAUME I DE CASTELLÓ

HTTPS://ORCID.ORG/0000-0001-6986-4163

Artículo recibido el / Article received: 2021-03-29

Artículo aceptado el / Article accepted: 2021-07-31

RESUMEN: Las democracias occidentales viven un impulso populista. En este contexto, la consolidación de las tecnologías digitales juega un papel clave por sus potencialidades para difundir con mayor facilidad los mensajes populistas. Esta investigación examina cómo los actores políticos populistas europeos introducen en su estrategia discursiva en Twitter la exclusión de grupos, el euroescepticismo y la existencia de crisis y cómo la enmarcan con el objetivo de conocer si existen diferencias significativas respecto a su ideología. La muestra está compuesta por los mensajes compartidos en Twitter por cuatro partidos políticos populistas europeos (Podemos, Movimiento 5 Estrellas, Frente Nacional y UKIP) y sus respectivos líderes (Pablo Iglesias, Beppe Grillo, Marine Le Pen y Nigel Farage y Paul Nuttall) durante tres periodos temporales fuera de campañas electorales. En total se han analizado 9.128 mensajes. Los principales hallazgos muestran que, pese a no ser características inherentes al fenómeno populista, la narrativa de la crisis, la exclusión de colectivos y el

\footnotetext{
${ }^{1}$ Este artículo forma parte de los proyectos I+D con referencias CSO2017-88620-P, financiado por la Agencia Española de Investigación dentro del Plan Estatal de I+D+i, y UJI-B2020-14, financiado por la Universitat Jaume I dentro del Plan de promoción de la investigación 2020.
} 
euroescepticismo está presente en el discurso de todos los actores políticos populistas analizados en Twitter. Todos ellos basan su estrategia discursiva en la búsqueda de culpables con el objetivo de presentarse ante el pueblo como la única opción política capaz de defenderles y devolverles la soberanía que les ha sido arrebatada. En este contexto, la ideología resulta determinante.

Palabras clave: Populismo, Comunicación Política, Redes Sociales, Twitter, Europa

ABSTRACT: Western democracies live a populist momentum. In this context, the consolidation of the digital technologies plays a key role because of their possibilities to widespread easier the populist messages. This research examines how European populist political actors introduce in their discursive strategy on Twitter the exclusion of groups, the Euroscepticism and the existence of a crisis and how they frame it with the aim to know if there are significant differences regarding their ideology. The sample are composed by the messages shared by four European political parties (Podemos, 5 Stars Movement, National Front and UKIP) and their leaders (Pablo Iglesias, Beppe Grillo, Marine Le Pen and Nigel Farage and Paul Nuttall) during three temporary periods, out of electoral campaigns. 9,128 messages have been analyzed. The main findings show that, despite not being inherent characteristics of the populist phenomenon, the narrative of the crisis, the exclusion of groups and Euroscepticism is present in the discourse of all the populist political actors analyzed on Twitter. All of them base their discursive strategy looking for the guilty in order to introduce themselves to the people as the only political option capable of defending them and restoring the sovereignty that has been taken from them. In this context, the ideology is a key element.

Key words: Populism, Political Communication, Social Media, Twitter, Europe

\section{INTRODUCCIÓN}

El debate político de las últimas décadas ha estado marcado por el fenómeno populista. Algunos autores incluso han llegado a decir que las democracias occidentales se encuentran inmersas en un Zeitgeist populista (Mudde, 2004), es decir, un período dominado por el surgimiento y desarrollo de nuevos movimientos populistas, tanto de izquierdas como de derechas, que buscan romper el statu quo que ha prevalecido hasta momento (Gerbaudo, 2018). En este contexto, el éxito electoral de algunos partidos políticos populistas ha aumentado el interés por este fenómeno. En Italia, el Movimiento 5 Estrellas fue la opción política más votada en las elecciones de 2018 y llegó a formar gobierno con La Liga de Mateo Salvini, un partido populista de extrema derecha (Chiaramonte et al., 2018). En Francia, en las elecciones presidenciales de 2017, el Frente Nacional obtuvo 7,5 millones de votos, y pasó a la segunda vuelta, disputándole la victoria a Emmanuel Macron (Ivaldi, 2018). En España, Podemos, situado a la izquierda del tablero político, emergió en las Elecciones Europeas de 2014, y consolidó su fuerza política ganando 79 escaños en el Parlamento español en 2016 (Casero-Ripollés, Feenstra y Tormey, 2016; Casero-Ripollés, Sintes-Olivella y Franch, 2017). Y en Gran Bretaña, el UKIP fue uno de los grandes defensores de la celebración del Brexit y de la salida de la 
Unión Europea por parte del Reino Unido (Usherwood, 2019). Precisamente, su variedad es lo que hace más interesante este fenómeno, en el que encontramos diferencias notables no solo por lo que respecta a su ideología, sino también en cuanto a su formación, su trayectoria política o sus propuestas programáticas (Caiani y Graziano, 2016).

Investigaciones previas consideran que gran parte del éxito de los movimientos populistas está vinculado a su estrategia comunicativa y discursiva, especialmente en redes sociales (Jagers y Walgrave, 2007; de Vreese et al., 2018). En la década de los noventa, la televisión era el medio más utilizado por las opciones políticas populistas para difundir sus mensajes (Mazzoleni, 2008). Por lo general, aprovechaban la cobertura de los tabloides para obtener más visibilidad y aumentar su número de votantes (Mazzoleni, 2003). Sin embargo, en la actualidad las redes sociales se han convertido en una herramienta fundamental para ellos, ya que les permite comunicarse de manera directa, fácil y rápida con sus seguidores y movilizar a sus votantes sin necesidad de contar con un elevado número de recursos económicos (Araújo y Prior, 2021) y evitando el filtro impuesto por los medios de comunicación convencionales (Aalberg y de Vreese, 2017).

La literatura previa sobre populismo se centra en el estudio de la comunicación populista sobre las actitudes políticas (Matthes y Schmuck, 2017), el tipo de votantes de los partidos populistas (Rooduijn, 2018), su agenda y el interés que genera en los usuarios (Alonso-Muñoz y Casero-Ripollés, 2018; Alonso-Muñoz, 2020) o en una única característica del populismo como es el euroescepticismo (Ivaldi, 2018). Además, la mayoría de los trabajos analizan la estrategia comunicativa y discursiva del populismo en un único país (Biancalana, 2014; Goodwin, 2015), siendo minoritarios los estudios a nivel comparado (Alonso-Muñoz y Casero-Ripollés, 2020).

El objetivo principal de este artículo es conocer la presencia y el encuadre de tres elementos propios de la retórica populista en la estrategia discursiva en Twitter de los actores políticos populistas europeos. En concreto, se analiza la presencia de la narrativa de la crisis, la exclusión de grupos o minorías y el euroescepticismo en los mensajes de Twitter de cuatro partidos políticos populistas europeos y sus respectivos líderes.

\section{POPULISMO, UN CONCEPTO CONTROVERTIDO}

El populismo es un concepto muy controvertido. Se ha intentado definir en numerosas ocasiones, pero su naturaleza cambiante y la multitud de variables nacionales que intervienen, han dificultado esta tarea. En este sentido, algunos autores destacan la ambigüedad del concepto (Taguieff, 1997), la vaguedad de las definiciones anteriores (Canovan, 1999) o que el término es camaleónico y puede adaptarse a contextos totalmente distintos (Taggart, 2000). En este sentido, podemos diferenciar tres enfoques desde los que abordar el estudio de este fenómeno. El primero considera al populismo como una forma concreta de organización política basada en la presencia de un tipo específico de líder que asciende a su gobierno con el apoyo inmediato y directo del pueblo (Germani, 1978; Di Tella, 1997). Esta concepción del populismo ha sido la más utilizada por la literatura que habla de este fenómeno en América Latina.

El segundo enfoque entiende el populismo como un estilo comunicativo que los partidos políticos y los líderes utilizan para movilizar a las masas (Jagers y Walgrave, 2007). Mediante el uso de mensajes simplistas que apelan a las emociones, los actores populistas pueden presentarse no solo como algo nuevo, diferente al establishment, sino como actores que defienden al pueblo en su lucha contra las élites (Moffitt, 2016; de Vreese et al., 2018). Jagers y Walgrave (2007) distinguen cuatro tipos de populismos: (1) Populismo vacío (solo apelan al pueblo); (2) Populismo excluyente (apelan al pueblo y excluyen a grupos o minorías); (3) Populismo anti-elitista (apelan al pueblo y critican a 
las élites); y (4) Populismo completo (apelan al pueblo, critican a las élites y excluyen a ciertos grupos). Y el tercero es el enfoque ideacional, que define el populismo como un conjunto de ideas que dividen a la sociedad en dos grupos: el pueblo bueno y la élite corrupta (Rovira Kaltwasser y Taggart, 2016; Rooduijn y Akkerman, 2017). En este contexto, la política debe respetar la soberanía popular (Mudde, 2004).

Pese a que en todos los enfoques se considera que los elementos clave del fenómeno populista son la apelación al pueblo y la crítica a las élites, el estudio del populismo durante la última década ha mostrado la existencia de otras características que están ganando peso en la estrategia comunicativa de los actores políticos populistas europeos como la narrativa de la crisis, el euroescepticismo (Alonso-Muñoz y CaseroRipollés, 2020) o la exclusión de las minorías. En este último caso, algunos estudios detectan un estilo comunicativo inclusivo, que busca defender e integrar a los colectivos vulnerables, practicado por el populismo de izquierdas (Sintes-Olivella, Casero-Ripollés y Yeste-Piquer, 2020). Estos elementos, aunque presentes en el discurso de todos los partidos en mayor o menor medida, varían en función de variables como su ideología o el lugar de procedencia de estos actores, lo que hace que su estudio sea de gran utilidad para ampliar el conocimiento de este fenómeno político y social.

\subsection{NARRATIVA DE LA CRISIS, EXCLUSIÓN DE GRUPOS Y EUROESCEPTICISMO EN LA ESTRATEGIA COMUNICATIVA DE LOS ACTORES POPULISTAS}

El populismo se esfuerza por demostrar que la sociedad está en crisis (Taggart, 2000). Su objetivo es simplificar al máximo la realidad para radicalizar y polarizar el debate político. Busca obligar al oponente, especialmente a los partidos del establishment, a actuar y adoptar las soluciones que ellos proponen. Así, frente a la política actual que requiere negociación, consenso y tiempo para resolver un problema, los actores populistas abogan por acciones rápidas y de cortoplacistas, haciendo un uso instrumentalizado y utilitario de la política (Saward, 2011). La crisis económica ha actuado como un fuerte catalizador de los recientes fenómenos populistas, ayudando a su consolidación y a su crecimiento. Por ello, ha capitalizado la atención de estos actores durante los últimos años, colocando en el centro de sus críticas al sistema financiero, al capitalismo y la austeridad generada por los recortes (Moffit y Tormey, 2014; Rooduijn, 2014). En su lucha perpetua contra las élites corruptas, los populistas han centrado su discurso en los efectos negativos que han provocado en la vida de las personas la recesión financiera y las políticas de austeridad adoptadas por los gobiernos europeos (Wodak, 2015).

No obstante, la narrativa de la crisis no solo hace alusión a cuestiones económicas, sino también a las dificultades sociales, migratorias y de representación en las sociedades actuales (Moffit y Tormey, 2014). En este contexto, la crisis del sistema tradicional de partidos también se ha incorporado como un argumento importante para los actores populistas (Rendueles y Sola, 2015). Esta práctica es especialmente común entre los nuevos partidos y líderes populistas, que se presentan como representantes del cambio y reclaman una profunda regeneración democrática, para paliar los efectos negativos de la crisis institucional y también para eliminar las prácticas corruptas en el sistema (CaseroRipollés, Sintes-Olivella y Franch, 2017). Atendiendo a la literatura previa, se formula la siguiente pregunta de investigación:

PI1: ¿Existen diferencias respecto al tipo de narrativa de la crisis que los actores políticos populistas utilizan en sus mensajes en Twitter en función de su ideología?

La crisis migratoria que ha vivido Europa durante los últimos años también ha captado la atención de los partidos populistas. La literatura previa ha señalado cómo la 
idea de exclusión propuesta por los actores populistas no se refiere solo a la élite, sino también a otros grupos que, por razones étnicas, religiosas o económicas, no forman parte del pueblo y son considerados por los actores populistas como los otros (Panizza, 2005; Albertazzi y McDonnell, 2008). Así, mientras que en la escala social las élites están por encima, los otros se sitúan por debajo del pueblo (Abts y Rummens, 2007). Realizando esta exclusión, los actores populistas buscan generar un sentimiento de pertenencia al grupo y discriminar a los que están fuera (Casero-Ripollés, 2007; van Dijk, 1997; Reinemann et al., 2017).

La exclusión de estos grupos ha generado una retórica antiinmigración que, en algunos casos, ha derivado en un discurso del odio hacia grupos sociales como los musulmanes (Gagliardone et al., 2015; Fuchs, 2017). En este contexto, las demandas de un control más estricto de la inmigración, las polémicas opiniones sobre los inmigrantes o la solicitud de asilo son una constante en la retórica de los partidos populistas de derechas (Alonso-Muñoz y Casero-Ripollés, 2020). De acuerdo con lo descrito anteriormente, se formula la siguiente pregunta de investigación:

PI2: ¿Bajo qué encuadre presentan la exclusión de grupos o minorías en su estrategia comunicativa en Twitter los actores políticos populistas europeos?

Esta actitud hostil contra la inmigración se ha incrementado durante las últimas décadas como consecuencia de la crisis económica en Europa. Especialmente tras la crisis económica mundial, Europa ha sufrido una crisis de valores que ha provocado que muchos de sus ciudadanos cuestionen algunos de los elementos definitorios de la Unión Europea e incluso su permanencia a ella, aspecto que la mayoría de los partidos populistas han incluido en sus programas electorales (Vasilopoulou, 2018). Según Mudde (2007), los partidos populistas no están en contra de los principios fundacionales de la Unión Europea, sino en contra de quienes dirigen la institución. En otras palabras, el euroescepticismo se traduce en la crítica hacia políticas concretas y no hacia la propia institución. En este sentido, podemos diferenciar diferentes tipos de euroescepticismos en función a la dimensión a la que afecte (van Klingeren, Boomgaarden y de Vreese, 2013). Así, podemos destacar, por ejemplo, el euroescepticismo económico, común entre los populistas de izquierdas y de derechas (van Elsas, Hakhverdian y van der Brug, 2016) y el euroescepticismo cultural que se centra en cuestiones de soberanía nacional (Mammone, 2009; Gerbaudo, 2017, 2018) o en la distinción entre nosotros y ellos en referencia a los inmigrantes (Kriesi, 2007; Leconte, 2010). Sin embargo, hay autores que sostienen que todas las dimensiones del euroescepticismo están conectadas entre sí (McLaren, 2006). Si bien la literatura previa ha detectado que la presencia del euroescepticismo es más propia de los partidos populistas de derechas, es necesario conocer cómo es el discurso de los actores populistas de izquierdas respecto a este tema. Por tanto, se plantea la siguiente pregunta de investigación:

PI3: ¿Existen diferencias según la ideología sobre el discurso euroescéptico planteado por los actores políticos populistas europeos en Twitter?

\section{METODOLOGÍA}

Para responder a las preguntas de investigación planteadas en este trabajo se ha realizado un análisis de contenido tanto cuantitativo como cualitativo. El objetivo no es únicamente conocer la presencia que tiene la narrativa de la crisis, la exclusión de grupos y minorías y el euroescepticismo en la estrategia comunicativa de los actores políticos populistas europeos en Twitter, sino también conocer el encuadre de este tipo de mensajes para saber si existen diferencias en función de su ideología. 
La muestra de esta investigación está compuesta por los mensajes publicados en Twitter por cuatro partidos políticos populistas europeos y sus respectivos líderes durante tres periodos temporales que eluden los periodos oficiales de campaña electoral. En concreto, se han analizado los mensajes de Twitter publicados en mayo y noviembre de 2016 y marzo de 2017 por Podemos y Pablo Iglesias (España); Movimiento 5 Estrellas (M5E) y Beppe Grillo (Italia); Frente Nacional (FN) y Marine Le Pen (Francia); y el Partido por la Independencia del Reino Unido (UKIP), Nigel Farage (líder del partido hasta el 30 de noviembre de 2016) y Paul Nuttall (líder del 30 de noviembre de 2016 hasta el 9 de junio de 2017) (Reino Unido).

Se han considerado tres criterios para la selección de la muestra. Primero, el eje ideológico. Podemos es considerado un partido populista de izquierdas y el Movimiento 5 Estrellas de centro, mientras que el Frente Nacional y el UKIP se ubican en la extrema derecha. En segundo lugar, el eje viejo-nuevo, considerando la trayectoria de los partidos políticos. Podemos y el Movimiento 5 Estrellas son partidos políticos de nueva creación, nacidos en 2014 y 2009 respectivamente, cuyo surgimiento está vinculado a las redes sociales (Rodríguez-Aguilera de Prat, 2015; Casero-Ripollés, Feenstra y Tormey, 2016). Por el contrario, el Frente Nacional (fundado en 1972 y refundado como Rassemblement National en 2018) y el UKIP (1993) tienen una trayectoria mucho más extensa en el tiempo. Finalmente, en tercer lugar, se trata de opciones políticas que provienen de cuatro países cuya relevancia política a nivel europeo es similar. Según datos del Fondo Monetario Internacional, Francia, Reino Unido, Italia y España son cuatro de las principales potencias europeas.

La muestra se descargó mediante la aplicación web Twitonomy, que en su versión premium permite descargar los tuits, retuits y respuestas de las cuentas de Twitter seleccionadas. Se recogieron un total de 18.739 mensajes, de los cuales se analizan 9.128 (2.348 pertenecen a los líderes y 6.780 a los partidos), que incluyen tanto los tuits como las respuestas (Tabla 1). Los retuits han sido descartados en esta investigación porque su objetivo es redistribuir información publicada por otros usuarios (Larsson, 2015) y, por lo tanto, no resultan de utilidad para dar respuesta a las preguntas de investigación planteadas en este trabajo. Hay que señalar que, con el objetivo de que el bajo número de publicaciones de Paul Nuttall no distorsionara los resultados y habiendo observado que la estrategia comunicativa que empleaba era la misma, los resultados de Paul Nuttall y Nigel Farage han sido agregados y se presentan de forma conjunta en la sección de resultados. Para el análisis de los datos se empleó el paquete estadístico SPSS (v.25). 
Tabla 1. Distribución de la muestra analizada

\begin{tabular}{|l|l|c|}
\hline \multirow{4}{*}{ Partidos } & & Número de mensajes analizados \\
\hline \multirow{4}{*}{ Líderes } & Podemos & 4.025 \\
\cline { 2 - 3 } & Movimiento 5 Estrellas & 280 \\
\cline { 2 - 3 } & Frente Nacional & 1.787 \\
\cline { 2 - 3 } & UKIP & 688 \\
\cline { 2 - 3 } & Total & 6.780 \\
\hline \multirow{5}{*}{} & Pablo Iglesias & 563 \\
\cline { 2 - 3 } & Beppe Grillo & 300 \\
\cline { 2 - 3 } & Nigel Farage & 1.229 \\
\cline { 2 - 3 } & Paul Nuttall & 211 \\
\cline { 2 - 3 } & Total & 45 \\
\hline & TOTAL & 2.348 \\
\hline
\end{tabular}

\section{RESULTADOS}

\subsection{LA NARRATIVA DE LA CRISIS EN EL DISCURSO POPULISTA EUROPEOS EN TWITTER}

Para simplificar la realidad, el populismo se esfuerza en demostrar que la sociedad está en crisis (Taggart, 2000). Su objetivo es polarizar a la ciudadanía para que tenga que posicionarse con la élite, corrupta y egoísta, o con ellos, los máximos representantes del pueblo. La narrativa de la crisis está presente en la estrategia comunicativa en Twitter de todos los actores políticos populistas analizados (PI1). En el caso de los dirigentes, destacan especialmente las cuentas de Beppe Grillo (63,67\%), y Nigel Farage y Paul Nuttall $(57,81 \%)$ que presentan valores muy elevados. Entre los partidos, los valores oscilan entre el 39,39\% del UKIP y el 71,62\% de Podemos. En general, todos los actores políticos populistas analizados coinciden en la existencia tanto de una crisis política como de una crisis económica.

Pablo Iglesias centra su discurso en la existencia de una crisis política $(39,47 \%)$ y social $(27,63 \%)$, ambas basadas en la creencia de que la corrupción política está afectando gravemente a la sociedad (Tabla 2). El líder de Podemos vincula los numerosos casos de corrupción que afectan al Gobierno español a la existencia de una profunda crisis política que sacude los cimientos de la democracia. Asimismo, considera que la irrupción de Podemos y sus políticas de regeneración están propiciando un profundo cisma que está rompiendo las bases del bipartidismo en España. Por otro lado, Iglesias hace una narrativa contundente de la crisis social que afecta a España (Tabla 2), en la que destaca la pobreza y la desigualdad que padecen los ciudadanos. Podemos, por su parte, centra su discurso en la creencia de que España está sumida en una profunda crisis política $(83,07 \%)$ marcada por los casos de corrupción del Partido Popular y la incertidumbre política que 
existe en Cataluña (Tabla 2). Respecto a la primera de las causas, Podemos acusa al Partido Popular de saquear los fondos públicos al tiempo que pide a los ciudadanos que asuman políticas de austeridad económica. Esta cifra está estrechamente relacionada con la presencia de una crisis económica (6,69\%), iniciada en 2008 (Tabla 2). En cuanto a la crisis política en Cataluña, Podemos apunta a la necesidad de diálogo. Consideran que el Gobierno debe apostar por implementar soluciones democráticas y no negarse a hablar con los partidos políticos independentistas.

Tabla 2. Narrativa de la crisis empleada por los actores políticos populistas analizados (\%)

\begin{tabular}{|c|c|c|c|c|c|c|c|c|}
\hline & Podemos & $\begin{array}{c}\text { P. } \\
\text { Iglesias }\end{array}$ & M5E & $\begin{array}{c}\text { B. } \\
\text { Grillo }\end{array}$ & FN & $\begin{array}{c}\text { M. } \\
\text { Le Pen }\end{array}$ & UKIP & $\begin{array}{c}\text { N. Farage + P. } \\
\text { Nuttall }\end{array}$ \\
\hline Crisis política & 83,07 & 39,47 & & 11,10 & 29,78 & 2,44 & 82,66 & 4,73 \\
\hline $\begin{array}{c}\text { Crisis } \\
\text { institucional }\end{array}$ & & 6,58 & 16,54 & & 5,62 & 9,15 & & 4,05 \\
\hline $\begin{array}{c}\text { Crisis } \\
\text { económica }\end{array}$ & 6,69 & 2,63 & & 7,41 & 30,90 & 37,20 & 1,10 & 7,43 \\
\hline $\begin{array}{c}\text { Crisis de } \\
\text { valores }\end{array}$ & 1,95 & 19,74 & 83,46 & 78,84 & & 2,44 & & \\
\hline $\begin{array}{c}\text { Crisis social } \\
\text { Crisis de } \\
\text { seguridad }\end{array}$ & 8,29 & 27,63 & & 2,65 & & & & \\
\hline $\begin{array}{c}\text { Crisis } \\
\text { migratoria }\end{array}$ & & 3,95 & & & 19,66 & 34,15 & 16,24 & 21,63 \\
\hline Crisis global & & & & & & & & 62,16 \\
\hline Otros & 100 & 100 & 100 & 100 & 100 & 100 & 100 & 100 \\
\hline TOTAL & 100,04 & 8,54 & & \\
\hline
\end{tabular}

Los populistas franceses son los que hacen una narrativa de la crisis más amplia, destacando especialmente la existencia de una crisis económica (30,90\% el FN y 37,20\% M. Le Pen) y política $(29,78 \%$ el FN y $2,44 \%$ M. Le Pen) y una crisis migratoria $(19,66 \%$ el FN y $34,15 \%$ M. Le Pen) sin precedentes (Tabla 2). En primer lugar, señalan la importancia de la crisis económica que vive Francia tras la aplicación de las medidas de austeridad impuestas por la Unión Europea. Ante la servidumbre mostrada por el gobierno francés a los designios de Europa, los populistas franceses proponen como solución la aplicación de medidas que favorezcan el patriotismo económico. Es decir, incentivar que se priorice el consumo de productos provenientes de Francia y las importaciones paguen mayores impuestos. Esta crisis económica está ligada a la existencia de una profunda crisis política marcada por la falta de liderazgo del gobierno francés, que consideran cada vez más distorsionado. En segundo lugar, los populistas franceses también presentan una narrativa de la crisis basada en la inmigración y la inseguridad que genera entre los ciudadanos (Tabla 2). Tanto Marine Le Pen como el Frente Nacional utilizan el miedo para fomentar la creencia de que la inmigración solo crea problemas y no aporta ningún beneficio a la sociedad. En este sentido, incluso argumentan que la existencia de comunas, además de la inseguridad que provocan, podría desembocar en una «guerra civil». Este tipo de retórica, basadas en el discurso del odio hacia determinados colectivos promueve la exclusión de cualquier persona en función de su raza o religión, hecho que tiene graves consecuencias, como la falta de integración en la sociedad de los inmigrantes.

Para el UKIP, el Reino Unido vive una crisis política sin precedentes $(82,66 \%)$ marcada por el Brexit y los deseos de los ciudadanos de salir de Europa (Tabla 2). En este 
contexto, dan voz a todos aquellos británicos que no quieren seguir las reglas de la Unión Europea y quieren que su país prospere. Argumentan que la solución es promover el «patriotismo» en todos los ámbitos, desde el económico al social, un discurso similar al que emplea también el Frente Nacional. Los dirigentes del UKIP, en cambio, aunque plantean los mismos postulados que su partido, defienden que la crisis en Reino Unido es global $(62,16 \%)$ dado que afecta a distintos ámbitos, como el empleo, la industria, la educación o los servicios sociales. Sin embargo, ambos comparten la idea de la existencia de una grave crisis migratoria (Tabla 2), en la que vinculan la inmigración con el aumento de casos de terrorismo en el Reino Unido mediante el uso de una retórica basada en el miedo al diferente. La solución para los populistas británicos es salir de la Unión Europea para que el Reino Unido no tenga que acoger a más inmigrantes.

Los populistas italianos, en cambio, presentan una narrativa de la crisis bastante diferente a la del resto de actores políticos populistas analizados. Consideran que Italia sufre una profunda crisis de valores (83,46\% el M5S y 78,84\% B. Grillo) (Tabla 2). Así lo demuestran los escritos publicados en el blog personal de Beppe Grillo, líder del Movimiento 5 Estrellas, que vincula los casos de corrupción a la falta de valores de la clase política como, por ejemplo, la honestidad. Destaca especialmente la falta de ética y moral que tienen los miembros del Partido Demócrata y su líder, Matteo Renzi, a quienes acusan de ser «ladrones de la democracia». En este contexto tan desfavorable para los italianos, Grillo y el Movimiento 5 Estrellas se postulan como una revolución que renovará las instituciones y la democracia, y como los únicos que pueden restablecer la soberanía del pueblo italiano.

\subsection{LA RETÓRICA ANTIINMIGRACIÓN EN EL DISCURSO POPULISTA EN TWITTER}

El rechazo a grupos o minorías por motivos étnicos o religiosos es una de las características que ha definido a la mayoría de los partidos y dirigentes populistas de derechas. De manera general, esta característica únicamente se ha encontrado en la estrategia comunicativa en Twitter del Frente Nacional y del UKIP y sus respectivos líderes (PI2).

Los actores políticos populistas de derechas presentan un discurso muy beligerante con la inmigración y, especialmente, con los musulmanes. Para ellos, los inmigrantes no son parte del pueblo, sino que representan a los otros, un colectivo que busca arrebatar los derechos a la ciudadanía y se configuran como una amenaza. A pesar de la disparidad que caracteriza a la inmigración, tanto el Frente Nacional como el UKIP la presentan como algo homogéneo contra la que el pueblo debe luchar si quiere mantener sus derechos. De esta forma, ambos actores políticos populistas vinculan la inmigración con la falta de oportunidades laborales o con la reducción de beneficios sociales que sufren sus respectivos países. Su objetivo es generar una representación negativa de la inmigración en el imaginario colectivo tanto del pueblo francés como del británico. La activación del eje amigo/enemigo (Schmitt, 2005) busca fomentar el enfrentamiento, aspecto que puede derivar en el rechazo, incluso violente, de los migrantes.

Tanto el Frente Nacional y el UKIP como sus dirigentes vinculan de forma clara y directa la inmigración con el terrorismo, mostrando un gran escepticismo sobre la gestión de la crisis migratoria que está llevando a cabo la Unión Europea y defendiendo la gestión que realizan países como Rusia. Ambas formaciones populistas consideran que junto parte de las personas que abandonan Siria por la guerra civil que está viviendo el país son terroristas del Estado Islámico, que aprovechan los beneficios humanitarios para cruzar las fronteras europeas y cometer atentados terroristas. El UKIP llega incluso a compartir un mensaje en el que argumenta que dos de los terroristas que perpetuaron los 
atentados de París en noviembre de 2015 entraron a Europa por las islas griegas tras estar registrados en un campo de refugiados de Turquía. Y el Frente Nacional considera que está comprobado que, a más inmigración, más atentados terroristas, hecho que «no les parece razonable». Nuevamente, con este tipo de mensajes ambos partidos populistas pretenden generar miedo e inseguridad entre los ciudadanos y presentarse como la única opción política capaz de proteger a los ciudadanos de la inmigración y sus consecuencias.

Los populistas italianos presentan una retórica equidistante ante algunos colectivos y minorías, ya que ni los critican ni los defienden abiertamente en sus mensajes de Twitter. Sin embargo, emplean una retórica en la que diferencian muy claramente a los ciudadanos italianos de los inmigrantes, aspecto que también busca remarcar las diferencias entre aquellas personas que tienen la ciudadanía italiana y, por lo tanto, poseen los derechos civiles que ésta conlleva, y aquellos que no la tienen, los inmigrantes, que no son considerados como parte del pueblo.

Por otro lado, Podemos y Pablo Iglesias muestran abiertamente su apoyo a grupos como el LGTBI o el colectivo transgénero, a los que agradecen su lucha por la igualdad y sus derechos. Practican, así, un populismo inclusivo con las minorías y los colectivos vulnerables. Esto concuerda con lo detectado por investigaciones previas que han identificado un estilo comunicativo inclusivo, practicado por el populismo de izquierda en las redes sociales, que persigue defender a los colectivos sociales más débiles (SintesOlivella, Casero-Ripollés y Yeste-Piquer, 2020). De la misma forma critican duramente aquellas acciones que perjudican a estos grupos, como sucedió con el autobús xenófobo Hazte Oír. En este sentido, aunque con estrategias muy diferentes, los populistas italianos y españoles rechazan el discurso xenófobo y homófobo, más propio de los partidos populistas de derechas.

\subsection{EL EUROESCEPTICISMO EN EL DISCURSO POPULISTA EN TWITTER}

La presencia de la retórica euroescéptica, en mayor o menor medida, está presente en la estrategia comunicativa en Twitter de todos los actores populistas analizados. No obstante, esta retórica gana fuerza en los mensajes compartidos por los actores políticos populistas de derechas como el Frente Nacional o el UKIP. En este sentido, encontramos dos tipos de estrategias (PI3) en función de la ideología.

Por un lado, los populistas españoles e italianos se muestran molestos con algunas acciones ejecutadas por la Unión Europea. En particular, su queja proviene de las políticas de austeridad económica que Europa ha exigido a los gobiernos nacionales (Tabla 3). Según su discurso, este tipo de políticas han resultado «altamente ineficientes» y lo único que han logrado ha sido empobrecer a la ciudadanía. En esta línea, el Movimiento 5 Estrellas defiende que Europa en la actualidad está a un paso del «punto de no retorno».

Aunque descontentos con el funcionamiento de esta institución, tanto Podemos como el Movimiento 5 Estrellas, apuestan por una redefinición de la Unión Europea en la que la ciudadanía juegue un papel más relevante y pueda participar de la toma de decisiones que les afectan. El Movimiento 5 Estrellas y su líder defienden que únicamente a través de la «democracia directa» será posible solucionar la crisis de credibilidad que vive la Unión Europea hoy. Podemos y Pablo Iglesias, por su parte, creen que es necesario actuar para recuperar los valores fundacionales de la Unión Europea: democracia, paz, libertad y solidaridad entre Estados. En esta línea, se muestran contrarios a algunos tratados económicos como el CETA, el tratado de libre comercio negociado por la Unión Europea y Canadá, que Podemos considera injusto. 
Tabla 3. Críticas a la Unión Europea en la estrategia comunicativa de los actores políticos populistas analizados $(\%)$

\begin{tabular}{|c|c|c|c|c|c|c|c|c|}
\hline & Podemos & $\begin{array}{c}\text { P. } \\
\text { Iglesias }\end{array}$ & M5E & $\begin{array}{c}\text { B. } \\
\text { Grillo }\end{array}$ & FN & $\begin{array}{c}\text { M. } \\
\text { Le Pen }\end{array}$ & UKIP & $\begin{array}{c}\text { N. Farage + P. } \\
\text { Nuttall }\end{array}$ \\
\hline $\begin{array}{c}\text { Políticas } \\
\text { migratorias }\end{array}$ & 3,03 & 33,33 & 9,09 & & 30,67 & 24,81 & 8,96 & 10,07 \\
\hline $\begin{array}{c}\text { Políticas } \\
\text { energéticas }\end{array}$ & 3,03 & & & & & & & \\
\hline $\begin{array}{c}\text { Políticas } \\
\text { económicas }\end{array}$ & 63,64 & 55,56 & 45,45 & 61,54 & 14,22 & 36,43 & 7,45 & 2,88 \\
\hline $\begin{array}{c}\text { Pérdida de } \\
\text { soberanía }\end{array}$ & 9,09 & 11,11 & 9,09 & 15,38 & 28,44 & 15,50 & 2,24 & 10,79 \\
\hline $\begin{array}{c}\text { Políticas } \\
\text { agrarias }\end{array}$ & & & & & 3,56 & 3,88 & 1,12 & \\
\hline $\begin{array}{c}\text { Políticas } \\
\text { antiterroristas }\end{array}$ & & & 4,55 & 23,08 & 3,56 & 1,55 & 2,99 & \\
\hline $\begin{array}{c}\text { Pérdida de } \\
\text { sentido de la UE }\end{array}$ & 9,09 & 18,18 & & 3,56 & 1,55 & 3,73 & \\
\hline $\begin{array}{c}\text { Abandono de la } \\
\text { UE }\end{array}$ & & 13,64 & & 6,21 & 12,40 & 73,51 & 76,26 \\
\hline $\begin{array}{c}\text { Abandono del } \\
\text { euro }\end{array}$ & & & & & 9,78 & 3,88 & & \\
\hline Otros & 12,12 & & & & & & & \\
\hline TOTAL & 100 & 100 & 100 & 100 & 100 & 100 & 100 & 100 \\
\hline
\end{tabular}

Por otro lado, los populistas franceses y británicos muestran una actitud mucho más crítica hacia la Unión Europea en sus discursos en Twitter. Los franceses son especialmente beligerantes con las políticas migratorias llevadas a cabo en Europa después de la crisis de los refugiados (Tabla 3). Ironizan con el hecho de que, al no tener fronteras, todos los inmigrantes que decida la canciller alemana Angela Merkel pueden entrar en Francia. Vinculados a los mensajes de crítica a las políticas migratorias están los que denuncian la pérdida de soberanía (Tabla 3). En este tipo de mensajes, afirman que los gobiernos nacionales han perdido el margen de acción en esta situación, que sólo depende de lo que decidan unos pocos privilegiados. El Frente Nacional considera que es necesario refundar Europa para que sea más respetuosa con los pueblos que la integran. Es decir, aspira a que la institución supranacional considere las particularidades de cada uno de los Estados miembros y que no legislen únicamente en beneficio de uno de los miembros, sino del conjunto. En algunas ocasiones, incluso plantean la posibilidad de salir de la Unión Europea y abandonar el euro para promover el «patriotismo económico y social» (Tabla 3 ).

En la misma línea están los mensajes publicados en Twitter por los populistas británicos, que presentan valores muy elevados en cuanto a la necesidad de salir de la Unión Europea (Tabla 3). Tanto el UKIP como sus líderes defienden la idea de que Reino Unido estará mejor fuera de Europa que dentro porque las políticas implementadas por la institución comunitaria perjudican gravemente al pueblo británico. Además de las políticas económicas, destaca especialmente su desacuerdo con las políticas migratorias (Tabla 3). En este sentido, los populistas británicos consideran que son muy perjudiciales para el pueblo británico porque la libre circulación de personas hace que los inmigrantes ocupen puestos de trabajo que deberían ocupar los ciudadanos británicos. En los mensajes que giran en torno a esta idea, el UKIP y sus dirigentes argumentan que tras abandonar la Unión Europea los ciudadanos británicos ganarán 200.000 nuevos puestos de trabajo. Los populistas británicos también señalan la notable pérdida de soberanía que genera la 
pertenencia a la Unión Europea (Tabla 3), argumentando que deben acatar las leyes aprobadas por el Parlamento Europeo, aunque no estén de acuerdo con ellas. En este sentido, el UKIP se muestra ante la ciudadanía como la única opción política que lucha contra las injusticias que se llevan a cabo en Europa, erigiéndose como el salvador y defensor de la soberanía nacional del pueblo británico.

Por tanto, observamos cómo los populistas españoles e italianos no se plantean salir de la Unión Europea, pero cuestionan algunas de las prácticas y políticas que se están llevando a cabo en la actualidad. Sin embargo, siempre lo hacen desde un punto de vista constructivo, sugiriendo propuestas alternativas para la mejora de esta institución. Por el contrario, los populistas de derechas (franceses e ingleses) optan por un discurso destructivo sobre la Unión Europea, con el que no buscan mejorarla, sino deslegitimarla y abandonarla para recuperar la soberanía nacional y aplicar, entre otros aspectos, medidas restrictivas contra la inmigración.

\section{CONCLUSIONES Y DISCUSIÓN}

Esta investigación nos ha permitido comprobar que la narrativa de la crisis, la exclusión de grupos y minorías y el euroescepticismo están presentes, en mayor o menor grado, en la estrategia discursiva en Twitter de todos los actores políticos populistas europeos analizados. Todos ellos basan su discurso en la búsqueda e identificación de culpables para presentarse ante el pueblo como la única opción viable si quieren recuperar la soberanía perdida. No obstante, se han detectado diferencias respecto al encuadre que le dan a estos mensajes los actores populistas en función de su ideología.

Respondiendo a la PI1, los datos demuestran que todos los actores políticos populistas analizados basan su estrategia discursiva en Twitter en denunciar la existencia de una profunda crisis. A pesar de sus diferencias ideológicas, comparten un discurso que destaca los efectos económicos negativos de la recesión y las políticas de austeridad adoptadas por sus respectivos gobiernos en la vida actual de las personas (Wodak, 2015). Sin embargo, como ya habían apuntado otras investigaciones al respecto (Moffitt y Tormey, 2014), los resultados muestran como también señalan la existencia de otros tipos de crisis más allá de la económica, cuya focalización y construcción discursiva varía en función de la ideología de cada partido.

Los populistas de centro y de izquierdas subrayan la existencia de una fuerte crisis política, social, institucional y de valores. Destacan la necesidad de una regeneración democrática que reprima la corrupción instalada en las instituciones de España e Italia. Solo así se podrán paliar los efectos negativos de la crisis institucional y del sistema de partidos (Rendueles y Sola, 2015). También denuncian una crisis de valores, que empuja a la clase política a corromperse por su falta de honestidad. Por otro lado, los populistas de derechas promocionan un discurso que se focalizan en la existencia de una fuerte crisis migratoria, que ha desencadenado en una crisis de seguridad nacional. Vinculan, así, inmigración y terrorismo. De esta forma, su discurso genera inseguridad en la sociedad, lo que provoca hostilidad y odio hacia los inmigrantes y los contrapone al pueblo, haciendo una clara distinción entre nosotros y ellos (van Dijk, 1997).

En relación con la idea anterior, la exclusión de grupo o minorías solo está presente en la estrategia comunicativa de los actores políticos populistas de derechas, (PI2). En este sentido, los partidos y líderes populistas franceses y británicos muestran una actitud hostil hacia la inmigración en general y los musulmanes en particular (Jagers y Walgrave, 2007). En sus mensajes de Twitter se puede observar una fuerte retórica antiinmigración (Wodak, 2015; Fuchs, 2017), utilizando el discurso de odio y vinculando la inmigración con el auge del terrorismo en Europa. Este colectivo es considerado el 
enemigo externo contra el que hay que combatir, activando la distinción amigo-enemigo señalada por Carl Schmitt (2005). Al presentar a los inmigrantes como los otros, buscan desvincularlos del pueblo y mostrarlos de forma negativa ante el imaginario colectivo (Reinemann et al., 2017). Considerándolos como una amenaza, justifican la implementación de duras medidas policiales contra este colectivo (Casero-Ripollés, 2007).

Respecto a los actores populistas de centro y de izquierdas el análisis detecta la existencia de dos estrategias distintas. Por un lado, los populistas españoles presentan un discurso inclusivo en defensa de grupos sociales débiles o vulnerables (Sintes-Olivella, Casero-Ripollés y Yeste-Piquer, 2020). Defienden abiertamente a los colectivos LGBTI y a las personas transgénero, y se muestran cercanos a los inmigrantes y exigen recuperar algunos derechos perdidos por este colectivo, como el acceso a la sanidad pública universal gratuita. Por otro lado, los populistas italianos presentan un discurso equidistante respecto a otros grupos, ya que ni los acusan ni los defienden. Sin embargo, en sus mensajes realizan una clara distinción entre ciudadanos e inmigrantes, lo que denota un cierto rechazo a la inmigración. En este sentido, aunque no critican abiertamente a este colectivo, también lo catalogan dentro de los otros y lo diferencian del pueblo.

Con relación a la PI3, el descontento con la Unión Europea está presente, a distintos niveles, en la estrategia discursiva en Twitter de todos los actores políticos populistas analizados, aunque en mayor medida en los mensajes de los populistas franceses y británicos. En términos generales, todos coinciden en resaltar la pérdida de soberanía que representa formar parte de la Unión Europea como un problema, porque obliga a los Estados miembros a cumplir con políticas migratorias, económicas y sociales que benefician a unos y perjudican a otros. La globalización, encarnada por organismos supranacionales como la Unión Europea, resta soberanía a los pueblos (Mammone, 2009), mientras que el Estado pierde parte del control para legislar a juicio de los populistas. En este punto, en línea con lo planteado por autores como Paolo Gerbaudo (2017), el tema de la soberanía es el vínculo entre el populismo tanto de izquierdas como de derechas. La diferencia entre ambos radica en cómo plantean su recuperación (Gerbaudo, 2018).

Los populistas de derechas presentan en Twitter un discurso destructivo en el que la única solución que plantean es salir de la Unión Europea. Para ellos, la recuperación de la soberanía nacional pasa por cerrar las fronteras para evitar la entrada de inmigrantes que ponen en riesgo tanto la seguridad nacional, por su vinculación con el terrorismo, como la cohesión social, por su falta de integración y adaptación a las costumbres religiosas y culturales de los países de acogida. En cambio, los populistas de centro y de izquierdas plantean en Twitter un discurso constructivo: no quieren salir de la Unión Europea, pero sí mejorar su posición dentro de ella y abrir un debate sobre su reformulación (Alonso-Muñoz y Casero-Ripollés, 2020). En este sentido, plantean la soberanía nacional como un tema social y democrático que implica lograr la igualdad de los pueblos, con el objetivo de que sus intereses prevalezcan sobre los de las élites. El pueblo debe tener el poder de manera real y ser decisivo en la toma de decisiones políticas. Sin embargo, a pesar de sus diferencias, todos coinciden en la necesidad de realizar referéndums para que el pueblo decida si quiere seguir formando parte de la Unión Europea o del euro como hasta ahora, o si quiere renovar o mejorar algún aspecto. Esta promesa de democracia directa es inherente a los desafíos antisistema del populismo y es lo que realmente los distingue de las formas extremistas no democráticas (Mammone, 2009).

Estos hallazgos permiten ampliar el conocimiento sobre el fenómeno populista desde una perspectiva comparada. Además, demuestran cómo, a pesar de no ser 
características inherentes del populismo, la narrativa de la crisis, la exclusión de los otros y el euroescepticismo están presentes en la estrategia discursiva y comunicativa de estos actores políticos. Asimismo, también aportan evidencias que la ideología es un factor capaz de condicionar la construcción discursiva de estos tres componentes en la política populista.

\section{REFERENCIAS BIBLIOGRÁFICAS}

Abts, Koen y Stefan Rummens. 2007. «Populism Versus Democracy». Political Studies, 55(2): 405-424. doi:10.1111/j.1467-9248.2007.00657.x

Aalberg, Toril y Claes H. de Vreese. 2017. «Introduction: Comprehending Populist Political Communication». En Populist Political Communication in Europe, ed. Toril Aalberg, Frank Esser, Carsten Reinemann, Jesper Strömbäck y Claes H. de Vreese. Nueva York: Routledge.

Albertazzi, Daniele y Duncan McDonnell. 2008. «Introduction: The Sceptre and the Spectre». En Twenty-First Century Populism, eds. Daniele Albertazzi y Duncan McDonnell. Nueva York: Palgrave Macmillan.

Alonso-Muñoz, Laura. 2020. "The 'More is more' effect: a comparative analysis of the political agenda and the strategy on Twitter of the European populist parties»». European Politics and Society, 21(5): 505-519. doi:10.1080/23745118.2019.1672921

Alonso-Muñoz, Laura y Andreu Casero-Ripollés. 2018. «Communication of European populist leaders on Twitter: Agenda setting and the 'more is less' effect». El Profesional de la Información, 27(6): 1193-1202. doi: 10.3145/epi.2018.nov.03

Alonso-Muñoz, Laura y Andreu Casero-Ripollés. 2020. «Populism Against Europe in Social Media: The Eurosceptic Discourse on Twitter in Spain, Italy, France, and United Kingdom During the Campaign of the 2019 European Parliament Election». Frontiers in Communication, 5. doi:10.3389/fcomm.2020.00054

Araújo, Bruno y Elder Prior. 2021. «Framing Political Populism: The Role of Media in Framing the Election of Jair Bolsonaro». Journalism Practice, 15(2): 226-242. Doi:10.1080/17512786.2019.1709881

Biancalana, Cecilia. 2014. «Il populismo nell' era di internet. Retorica e uso del web nel Movimento 5 Stelle». Il Mulino, (1): 53-62. doi: 10.1402/75752

Caiani, Manuela y Paolo Graziano. 2016. «Varieties of Populism: Insights from the Italian Case». Rivista Italiana di Scienza Politica, 46(2): 243-267. doi:10.1017/ipo.2016.6

Canovan, Margaret. 1999. «Trust the People! Populism and the Two Faces of Democracy». Political Studies, 47(1): 2-16. doi:10.1111/1467-9248.00184

Casero-Ripollés, Andreu. 2007. «Inmigración e ilegalidad: la representación mediática del "otro" como problema». Cultura, Lenguaje y Representación, 4(4): 33-47.

Casero-Ripollés, Andreu, Marçal Sintes-Olivella y Pere Franch. 2017. «The Populist Political Communication Style in Action: Podemos's Issues and Functions on Twitter During the 2016 Spanish General Election». American Behavioral Scientist, 61(9): 986-1001. doi: 10.1177/0002764217707624

Casero-Ripollés, Andreu, Ramón A. Feenstra y Simon Tormey. 2016. «Old and New Media Logics in an Electoral Campaign. The Case of Podemos and the Two-Way Street Mediatization of Politics». The International Journal of Press/Politics, 21(3): 378-397. doi: 10.1177/1940161216645340

Chiaramonte, Alessandro, Vincenzo Emanuele, Nicola Maggini y Aldo Paparo. 2018. «Populist Success in a Hung Parliament: The 2018 General Election in Italy». 
South European Society and Politics, 23(4): 479-501. doi:10.1080/13608746.2018.1506513

de Vreese, Claes H., Frank Esser, Toril Aalberg, Carsten Reinemann y James Stanyer. 2018. «Populism as an expression of political communication content and style: A new perspective». The International Journal of Press/Politics, 23(4): 423-438. doi:10.1177/1940161218790035

Di Tella, Torcuato S. 1997. «Populism into the Twenty-first Century». Government and Opposition, 32(2): 187-200. doi:10.1111/j.1477-7053.1997.tb00157.x

Fuchs, Christian. 2017. «Donald Trump: A Critical Theory-Perspective on Authoritarian Capitalism». tripleC: Communication, Capitalism \& Critique, 15: 1-72. doi: 10.31269/triplec.v15i1.835

Gagliardone, Iginio, Danit Gal, Thiago Alves, y Gabriela Martínez. 2015. Countering Online Hate Speech. Paris: UNESCO Publishing.

Gerbaudo, Paolo. 2018. «Social media and populism: an elective affinity?». Media, Culture \& Society, 40(5): 745-753. doi: 10.1177/0163443718772192

Gerbaudo, Paolo. 2017. «The populist era». Soundings, 65: 46-58.

Germani, Gino. 1978. Authoritarianism, fascism, and national populism. New Brunswick: Transaction Books.

Goodwin, Matthew J. 2015. «UKIP, the 2015 General Election and Britain's EU Referendum». Parliamentary Insight, 6(3): 12-15. doi:10.1111/2041-9066.12107

Ivaldi, Gilles. 2018. «Contesting the EU in times of crisis: The Front National and politics of Euroscepticism in France». Politics, 38(3): 278-294. doi:10.1177/0263395718766787

Jagers, Jan y Stefaan Walgrave. 2007. «Populism as political communication style: An empirical study of political parties' discourse in Belgium». European Journal of Political Research, 46(3): 319-45. doi: 10.1111/j.1475-6765.2006.00690.x

Kriesi, Hanspeter. 2007. "The role of European integration in national election campaigns». European Union Politics, 8(1): 83-108. doi: $10.1177 / 1465116507073288$

Larsson, Anders-Olof. 2015. "Comparing to prepare: Suggesting ways to study social media today-and tomorrow». Social Media \& Society, 1(1): 1-2. doi:10.1177/2056305115578680

Leconte, Cécile. 2010. Understanding Euroscepticism. Basingstoke: Palgrave Macmillan.

Mammone, Andrea. 2009. «The eternal return? Faux populism and contemporarization of neo-fascism across Britain, France and Italy». Journal of Conemporary European Studies, 17(2): 171-192. doi: 10.1080/14782800903108635

Matthes, Jörg y Desirée Schmuck. 2017. «The effects of anti-immigrant right-wing populist ads on implicit and explicit attitudes: A moderated mediation model». Communication Research, 44(4): 556-581. doi:10.1177/0093650215577859

Mazzoleni, Gianpietro. 2008. «Populism and the media». En Twenty-First Century Populism: The Spectre of Western European Democracy, ed. Daniele Albertazzi y Duncan McDonnell. Basingstoke: Palgrave Macmillan

Mazzoleni, Gianpietro. 2003. "The Media and the Growth of Neo-Populism in Contemporary Democracies». En The Media and Neo-Populism, eds. Gianpietro Mazzoleni, Julianne Stewart y Bruce Horsfield. Londres: Praeger.

McLaren, Lauren M. 2006. Identity, Interests and Attitudes to European Integration. Basingstoke: Palgrave Macmillan

Moffitt, Benjamin. 2016. The global rise of populism: Performance, political style, and representation. Stanford: Stanford Univeristy Press. 
Moffitt, Benjamin y Simon Tormey. 2014. «Rethinking populism: Politics, mediatisation and political style». Political Studies, 62(2): 381-397. doi:10.1111/14679248.12032

Mudde, Cas. 2007. Populist Radical Right Parties in Europe. Cambridge: Cambridge University Press.

Mudde, Cas. 2004. «The Populist Zeitgeist». Government and Opposition, 39(3): 541563. doi: 10.1111/j.1477-7053.2004.00135.x

Panizza, Francisco. 2005. «Introduction: Populism and the Mirror of Democracy». En Populism and the Mirror of Democracy, ed. Francisco Panizza. Londres: Verso.

Reinemann, Carsten, Toril Aalberg, Frank Esser, Jesper Strömbäck y Claes H. de Vreese. 2017. «Populist Political Communication: Toward a Model of Its Causes, Forms, and Effects». En Populist Political Communication in Europe, eds. Toril Aalberg, Frank Esser, Carsten Reinemann, Jesper Strömbäck y Claes H. de Vreese. Nueva York: Routledge.

Rendueles, César y Jorge Sola. 2015. «Podemos y el "populismo de izquierdas”: ¿Hacia una contrahegemonía desde el sur de Europa?». Nueva Sociedad, 258: 29-44.

Rodríguez-Aguilera de Prat, Cesáreo. 2015. «Semejanzas y diferencias entre el Movimento 5 Stelle y Podemos». Società Mutamento Politica, 6(11): 51-74. doi:10.13128/SMP-16400

Rooduijn, Matthijs. 2018. «What unites the voter bases of populist parties? Comparing the electorates of 15 populist parties». European Political Science Review, 10(3): 351-368. doi:10.1017/S1755773917000145

Rooduijn, Matthijs. 2014. «The nucleus of populism: In search of the lowest common denominator». Government and Opposition, 49(4): 537-599. doi:10.1017/gov.2013.30

Rooduijn, Matthijs y Tjitske Akkerman. 2017. «Flank Attacks: Populism and Left Right Radicalism in Western Europe». Party Politics, 23(3): 193-204. doi:10.1177/1354068815596514

Rovira-Kaltwasser, Cristóbal y Paul Taggart. 2016. «Dealing with Populists in Government: A Framework for Analysis». Democratization, 23(2): 201-220. doi:10.1080/13510347.2015.1058785

Saward, Michael. 2011. «Slow Theory: Taking Time over Transnational Democratic Representation». Ethics and Global Politics, 4(1): 1-18. doi:10.3402/egp.v4i1.6416

Schmitt, Carl. 2005. Concepto de lo político. Madrid: Alianza.

Sintes-Olivella, Marçal, Andreu Casero-Ripollés y Elena Yeste-Piquer. 2020. «The Inclusionary Populist Communication Style on Facebook: The Case of Ada Colau in Barcelona». Communication \& Society, 33(2): 193-208. doi: 10.15581/003.33.2.193-208

Taggart, Paul. 2000. Populism. Concepts in the Social Sciences. Buckingham: Open University Press.

Taguieff, Pierre-André. 1997. «Le populisme et la science politique. Du mirage conceptuel aux vrais problems». Vingtieme siecle. Revue d'histoire, 56: 4-33. doi: $10.2307 / 3770596$

Usherwood, Simon. 2019. «Shooting the fox? UKIP's populism in the post-Brexit era». West European Politics, 1-21. doi:10.1080/01402382.2019.1596692

van Dijk, Teun A. 1997. Racismo y análisis crítico de los medios. Barcelona: Paidós.

van Elsas, Erika J., Armen Hakhverdian y Wouter van der Brug. 2016. «United against a common foe? The nature and origins of Euroscepticism among left-wing and right-wing citizens». West European Politics, 39(6): 1181-1204. 
van Klingeren, Marijn, Hajo G. Boomgaarden y Claes H. de Vreese. 2013. «Going soft or staying soft: have identity factors become more important than economic rationale when explaining euroscepticism?». Journal of European Integration, 35(6): 689-704. doi: 10.1080/07036337.2012.719506

Vasilopoulou, Sofia. 2018. «The radical right and Euroskepticism». En The Oxford Handbook of the Radical Right, ed. Jens Rydgren. Oxford: Oxford University Press.

Wodak, Ruth. 2015. The Politics of Fear: What Right-Wing Populist Discourses Mean. Londres: Sage. 